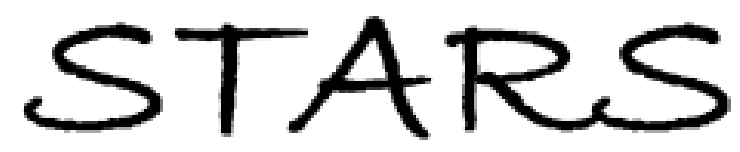

University of Central Florida

STARS

$1-1-2003$

\title{
Cathodoluminescence studies of the electron injection-induced effects in GaN
}

Leonid Chernyak

University of Central Florida

William Burdett

University of Central Florida

Mikhail Klimov

University of Central Florida

Andrei Osinky

Find similar works at: https://stars.library.ucf.edu/facultybib2000

University of Central Florida Libraries http://library.ucf.edu

This Article is brought to you for free and open access by the Faculty Bibliography at STARS. It has been accepted for inclusion in Faculty Bibliography 2000 s by an authorized administrator of STARS. For more information, please contact STARS@ucf.edu.

\section{Recommended Citation}

Chernyak, Leonid; Burdett, William; Klimov, Mikhail; and Osinky, Andrei, "Cathodoluminescence studies of the electron injection-induced effects in GaN" (2003). Faculty Bibliography 2000s. 3671.

https://stars.library.ucf.edu/facultybib2000/3671

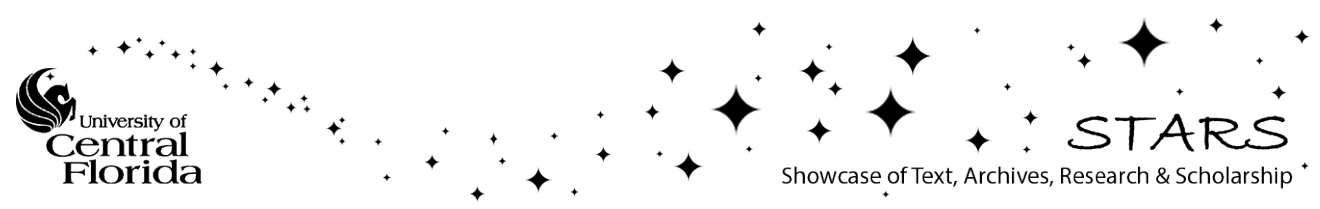




\section{Cathodoluminescence studies of the electron injection-induced effects in GaN}

Cite as: Appl. Phys. Lett. 82, 3680 (2003); https://doi.org/10.1063/1.1578514

Submitted: 06 February 2003. Accepted: 10 April 2003. Published Online: 20 May 2003

Leonid Chernyak, William Burdett, Mikhail Klimov, and Andrei Osinsky

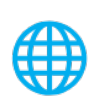

View Online

\section{ARTICLES YOU MAY BE INTERESTED IN}

Effects of excitation density on cathodoluminescence from GaN

Applied Physics Letters 79, 2154 (2001); https://doi.org/10.1063/1.1408273

Cathodoluminescence studies of carrier concentration dependence for the electronirradiation effects in $p-\mathrm{GaN}$

Applied Physics Letters 90, 172111 (2007); https://doi.org/10.1063/1.2733620

Luminescence properties of defects in $\mathrm{GaN}$

Journal of Applied Physics 97, 061301 (2005); https://doi.org/10.1063/1.1868059

\section{Applied Physics Letters}

Mid-IR and THz frequency combs special collection 


\title{
Cathodoluminescence studies of the electron injection-induced effects in GaN
}

\author{
Leonid Chernyak, ${ }^{\text {a) }}$ William Burdett, and Mikhail Klimov \\ Physics Department, University of Central Florida, Orlando, Florida 32816-2385 \\ Andrei Osinsky \\ SVT Associates, Eden Prairie, Minnesota 55344
}

(Received 6 February 2003; accepted 10 April 2003)

\begin{abstract}
Local irradiation of $p$-type $\mathrm{GaN}$ with the electron beam of a scanning electron microscope resulted in up to a threefold decrease of the peak cathodoluminescence intensity at $\sim 379 \mathrm{~nm}$, as was observed in the variable temperature measurements. The cathodoluminescence results are consistent with an increase of the minority carrier diffusion length in the material, as is evident from the electron-beam-induced current measurements. The activation energy for the electron injection effect, estimated from the temperature-dependent cathodoluminescence, is in agreement with the thermal ionization energy of the Mg-acceptor in GaN. (C) 2003 American Institute of Physics.
\end{abstract}

[DOI: $10.1063 / 1.1578514]$

Current applications of $\mathrm{GaN}$ and related compounds necessitate availability of high quality $p$ - and $n$-type material. Because of the difficulties associated with $p$-type doping, studies of the $\mathrm{Mg}$ acceptor in III-nitrides have attracted a lot of attention. It has been recently discovered that electron injection into $p-\mathrm{GaN}$ leads to a pronounced change in the material's transport properties. ${ }^{1-3}$ In particular, a multiplefold increase of the minority electron diffusion length, $L$, has been observed within $2000 \mathrm{~s}$ (at most) of sample irradiation with the electron beam of a scanning electron microscope (SEM) or of forward bias electron injection into a solid-state device ( $p-n$ junction or Schottky barrier). ${ }^{1-3}$ The increase of diffusion length was monitored in situ in the SEM using the electron-beam-induced current (EBIC) technique. ${ }^{1-5}$ Recent temperature dependent EBIC experiments ${ }^{4,5}$ attributed the observed electron injection-induced effect to the charging of Mg-related levels. The activation energy of the effect was found to be consistent with that for the thermal activation of Mg dopants.

Electron injection-induced changes in the minority carrier transport have a pronounced optical signature. Room temperature spectral photoresponse measurements, carried out on $\mathrm{GaN} p-n$ junctions before and after forward bias application, demonstrated a significant increase in peak responsivity at $\sim 365 \mathrm{~nm}$, due to an electron injection-induced enhancement of $L$ in the $p$-type region. ${ }^{6}$ This is because the increased diffusion length improves minority carrier collection and eliminates the "dead space," where carriers recombine before they are collected. ${ }^{7}$

In this letter we report variable temperature cathodoluminescence (CL) studies of bulk GaN before and after electron injection in the SEM. CL results are correlated with the electron injection-induced enhancement of $L$ and provide another experimental evidence for the relation of the phenomenon of interest to $\mathrm{Mg}$ doping.

Several bulk (2-3- $\mu$ m-thick) GaN samples grown by

${ }^{a)}$ Electronic mail: chernyak@physics.ucf.edu metalorganic chemical vapor deposition (MOCVD) on sapphire substrates were studied. These samples demonstrated a hole concentration in the mid $10^{17} \mathrm{~cm}^{-3}$ with a mobility of $\sim 7 \mathrm{~cm}^{2} / \mathrm{V} \mathrm{s}$. The experiments were carried out in situ in a Philips XL30 SEM, which is integrated with a Gatan MonoCL3 cathodoluminescence system, allowing wavedependent and temperature-dependent optical measurements. The sample temperatures in the CL measurements were varied from 20 to $200^{\circ} \mathrm{C}$. The emitted radiation was analyzed using a single grating (1200 lines $/ \mathrm{mm}$, blazed at $500 \mathrm{~nm})$ and a Hamamatsu photomultiplier tube, sensitive in the 185-850 $\mathrm{nm}$ range. For every temperature, CL measurements were carried out at a different location, which was continuously excited (up to $2200 \mathrm{~s}$ ) by a $30 \mathrm{keV}$ beam. The current, absorbed in the material under this excitation, corresponds to several $10 \mathrm{nA}$. The excitation was combined with a periodic acquisition of cathodoluminescence spectra, taken under a SEM magnification of $\times 10000$. CL results were compared with those obtained from the EBIC measurements, because the same samples were studied by both techniques. A detailed description of our EBIC experiments can be found elsewhere. $^{1-5}$

The experiments were started at room temperature with the initial CL spectrum acquisition (spectrum 1 in Fig. 1). This spectrum features a broad luminescence band, peaking at $\sim 379 \mathrm{~nm}(3.265 \mathrm{eV})$ and attributed to the excited electron transition from the conduction band to the $\mathrm{Mg}$-acceptorrelated levels $\left(e-\mathrm{Mg}^{0}\right){ }^{8}$ Figure 1 shows a continuous decay of CL intensity, as is observed in the spectra $1-5$. We attribute a decrease of luminescence intensity to an increase in the minority carrier (electron) lifetime, due to a charging of the neutral Mg levels in $p$-GaN. ${ }^{4,5}$ Note, that the concentration of these levels is quite significant (up to $10^{20} \mathrm{~cm}^{-3}$ ), because of the large values $(130-208 \mathrm{meV})^{9-11}$ of the thermal ionization energy for $\mathrm{Mg}$ acceptors. Trapping of the SEM-beam-injected electrons by the Mg level prevents recombination of the conduction band electrons (generated by the same beam) through it. This leads to an increase of minority electron lifetime in the conduction band. ${ }^{4,5}$ Because 


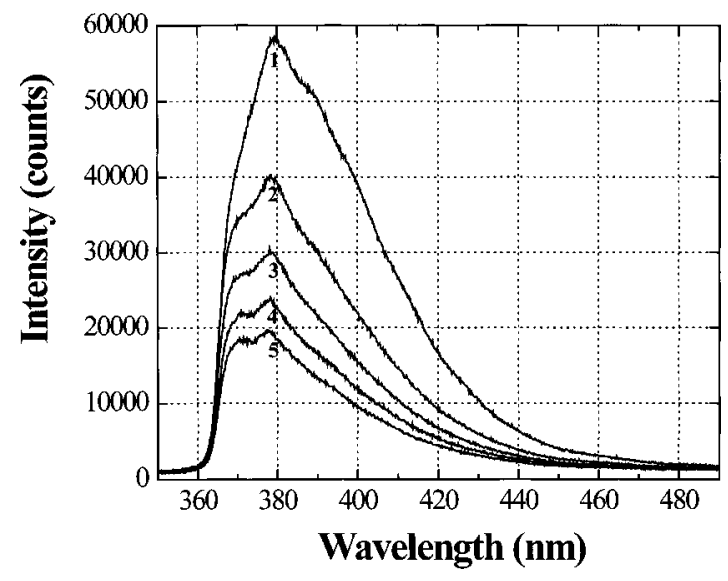

FIG. 1. Room temperature cathodoluminescence measurements after different time intervals of electron injection. The maximum $(379 \mathrm{~nm})$ in spectrum 1 nearly corresponds to the pre-injection situation (less than $20 \mathrm{~s}$ time difference). The maximum in spectra $2,3,4$, and 5 corresponds respectively to $660,1200,1680$, and $2200 \mathrm{~s}$ of electron injection.

the intensity of luminescence is proportional to the rate of radiative recombination, ${ }^{12}$ which decreases with increasing lifetime, the CL decay is observed in Fig. 1. We note that a $\sim 2 \mathrm{~nm}$ "blue" shift of the $e-\mathrm{Mg}^{0}$ peak, observed in Fig. 1 with increasing electron injection time, is possibly attributed to a continuous charging of additional $\mathrm{Mg}$ levels in the forbidden gap.

Increase of the minority electron lifetime, $\tau$, in $p$-GaN determines an increase of diffusion length for this carrier according to the following equation:

$$
L=\sqrt{D \tau}
$$

where $D$ is carrier diffusivity, which is unaffected by electron injection. ${ }^{1}$ Figure 2 shows the linear dependence of $L$ (open circles) on the time of electron injection for the sample under investigation. The minority electron diffusion length increases and saturates within $\sim 2000$ seconds of injection. ${ }^{1-5}$ The increase of $L$ is consistent with the decrease of the peak CL intensity (increase of $\tau$ ), which is also shown in Fig. 2 (open squares). Because $L$ depends linearly on the time of electron injection, the CL intensity (which is propor-

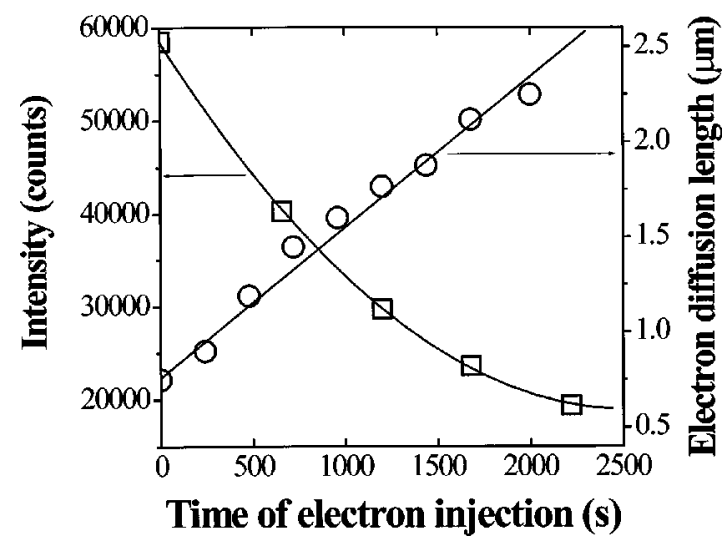

FIG. 2. Dependence of the maxima (open squares) for the room temperature CL spectra (Fig. 1) on the time of electron injection, and the quadratic fit. Also shown: dependence of the minority electron diffusion length (open circles) in the same sample on the time of electron injection, and the linear fit.

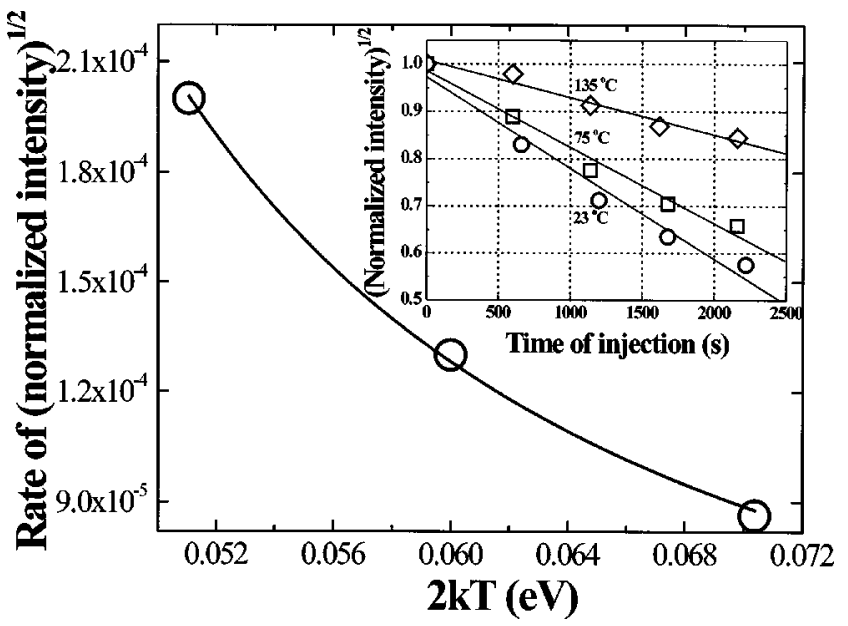

FIG. 3. Dependence for the rate of the square root of normalized intensity (open circles) on the thermal energy, and the exponential fit. Inset: Variable temperature dependence for the square root of normalized intensity on the time of electron injection. The rate at every temperature is obtained from the slope of a linear fit.

tional to $\tau^{-1}$ ) should depend on the injection time quadratically, in agreement with Eq. (1). This is, indeed, observed in Fig. 2.

CL measurements, carried out at elevated temperatures, demonstrated a slower decrease of the peak CL intensity with the time of electron injection. The rates, $R$, for this decrease were found from the dependencies of the square root of normalized (with respect to the initial maximum value) intensity on the time of electron injection (cf. inset of Fig. 3). In Fig. 3 , these rates are plotted as a function of $2 \mathrm{kT}$ and fitted (similar to what was previously done for the rates of $L)^{4,5}$ using the following equation:

$$
R=R_{0} \exp \left(\frac{\Delta E_{A}}{2 k T}\right) .
$$

Here, $R_{0}$ is the scaling constant, $\Delta E_{A}$ is the process activation energy, and $T$ is the temperature. The best fit for Eq. (2) was obtained when $\Delta E_{A}=153 \mathrm{meV}$. This is in good agreement with the activation energy of the electron injection effect, obtained for the same sample from the temperature dependent EBIC measurements $(178 \mathrm{meV}){ }^{5}$ This activation energy is also consistent with the thermal ionization energy for the $\mathrm{Mg}$ acceptor in $\mathrm{GaN} .{ }^{9-11}$

The Mg level, containing a trapped injected electron, becomes again available for recombination of the conduction band electrons as this level captures a hole. Capturing a hole means a transition of the trapped electron to the valence band. The rate of this transition increases with increasing temperature, ${ }^{13}$ and we note the existence of the activation energy for a hole capture. The latter activation energy is, in fact, $\Delta E_{A}$, obtained in this work.

As the rate of hole capture on the $\mathrm{Mg}$ level increases, the conduction band electrons have more chance for recombination through this level. This results in a shortening of the minority electron lifetime and a slower rate for the square root of CL intensity at higher temperatures (cf. inset of Fig. 3).

It has to be pointed out that the activation energy for a hole capture on the $\mathrm{Mg}$ level is generally lower than the 
thermal ionization energy for this level. However, the measurements of persistent photoconductivity in $p$ - $\mathrm{GaN}^{11,14} \mathrm{im}$ ply that these two activation energies may, actually, be quite close to each other, with the difference not exceeding 30 meV. This assumption was used in the interpretation of our experimental results.

It has to be also noted that no changes in CL intensity were observed in $n$-type GaN. This is another argument for the involvement of $\mathrm{Mg}$ levels in the phenomenon of electron injection. Because the electron injection-induced changes in minority carrier transport and optical photoresponse were previously observed in $p$-type molecular beam epitaxy (MBE) grown samples, ${ }^{1,5,6}$ the role of hydrogen in the effects of electron injection is excluded. This is since MBE GaN is largely hydrogen free. ${ }^{15}$

Finally, we would like to stress that while we do not completely exclude the role of electron beam-induced defects in the decrease of CL intensity (due to creation of nonradiative centers), we believe that their concentration is insufficient for a significant impact. In our previous work we have studied the influence of defect density on minority carrier diffusion length in thick hydride vapor-phase epitaxy grown GaN films. ${ }^{16}$ It has been found that the minority carrier diffusion length in these films shows a several-fold decrease with the defect density increasing from $10^{8}$ (typical for $\mathrm{GaN}$ ) to $10^{9} \mathrm{~cm}^{-2}$. Therefore, if a significant amount of defects was induced by an electron beam in this work, we would then expect a decrease in $L$, but not its increase as is seen in Fig. 2.

The impact of electron injection on GaN optical properties was investigated in bulk MOCVD-grown $p-\mathrm{GaN}$ at variable temperatures. The experimental results provided an additional evidence for the relation of the phenomenon of interest to the charging of $\mathrm{Mg}$ levels. The activation energy, obtained for the electron injection-induced effect from the CL measurements, is in agreement with that previously found in the temperature-dependent EBIC measurements.
Additional studies are under way to understand the kinetics of luminescence intensity relaxation to its pre-injection level, after an electron beam irradiation is stopped.

This research was supported in part by the University of Central Florida, through its in-house grant, the Petroleum Research Fund (PRF No. 38189-G5), and the National Science Foundation (ECS No. 0219546 and ECS No. 0216055).

${ }^{1}$ L. Chernyak, A. Osinsky, V. Fuflyigin, and E. F. Schubert, Appl. Phys. Lett. 77, 875 (2000)

${ }^{2}$ L. Chernyak, A. Osinsky, and A. Schulte, Solid-State Electron. 45, 1687 (2001)

${ }^{3}$ L. Chernyak, G. Nootz, and A. Osinsky, Electron. Lett. 37, 922 (2001).

${ }^{4}$ L. Chernyak, W. Burdett, and A. Osinsky, Appl. Phys. Lett. 81, 1633 (2002).

${ }^{5}$ W. Burdett, A. Osinsky, V. Kotlyarov, P. Chow, A. Dabiran, and L. Chernyak, Solid-State Electron. 47, 931 (2003).

${ }^{6}$ L. Chernyak, A. Schulte, A. Osinsky, J. Graff, and E. F. Schubert, Appl. Phys. Lett. 80, 926 (2002).

${ }^{7}$ D. J. H. Lambert, M. M. Wong, U. Chowdhury, C. Collins, T. Li, H. K. Kwon, B. S. Shelton, T. G. Zhu, J. C. Campbell, and R. D. Dupuis, Appl. Phys. Lett. 77, 1900 (2000).

${ }^{8}$ O. Gelhausen, H. N. Klein, M. R. Phillips, and E. M. Goldys, Appl. Phys. Lett. 81, 3747 (2002).

${ }^{9}$ A. Y. Polyakov, N. B. Smirnov, A. V. Govorkov, A. V. Osinsky, P. E. Norris, S. J. Pearton, J. Van Hove, A. M. Wowchack, and P. P. Chow, J. Appl. Phys. 90, 4032 (2001).

${ }^{10}$ W. Gotz, R. S. Kern, C. H. Chen, H. Liu, D. A. Steigerwald, and R. M. Fletcher, Mater. Sci. Eng., B 59, 211 (1999).

${ }^{11}$ J. Z. Li, J. Y. Lin, H. X. Jiang, A. Salvador, A. Botchkarev, and H. Morkoc, Appl. Phys. Lett. 69, 1474 (1996).

${ }^{12}$ J. I. Pankove, Optical Processes in Semiconductors (Prentice-Hall, Englewood Cliffs, NJ, 1971)

${ }^{13}$ S. M. Sze, Semiconductor Devices Physics and Technology (Wiley, New York, 1985)

${ }^{14}$ C. Johnson, J. Y. Lin, H. X. Jiang, M. A. Khan, and C. J. Sun, Appl. Phys. Lett. 68, 1808 (1996).

${ }^{15}$ M. A. Reshnikov, P. Visconti, and H. Morkoc, Appl. Phys. Lett. 78, 177 (2001)

${ }^{16}$ L. Chernyak, A. Osinsky, G. Nootz, A. Schulte, J. Jasinski, M. Benamara, Z. Liliental-Weber, D. C. Look, and R. J. Molnar, Appl. Phys. Lett. 77, 2695 (2000) 